\title{
JAK2 Inhibitor XL019
}

National Cancer Institute

\section{Source}

National Cancer Institute. AK2 Inhibitor XL019. NCI Thesaurus. Code C90573.

An orally bioavailable inhibitor of Janus-associated kinase 2 (JAK2) with potential

antineoplastic activity. XL019 inhibits the activation of JAK2 as well as the mutated form JAK2V617F, which may result in the inhibition of the JAK-STAT signaling pathway and may induce apoptosis. The JAK2 mutated form JAK2V617F has a valine-to-phenylalanine modification at position 617 and plays a key role in tumor cell proliferation and survival. 